\title{
Design on smart water meter system based on Cloud Computing
}

\author{
Xiye Feng ${ }^{1, *}$, Dawei Zang ${ }^{2}$, Lelin Yan ${ }^{1}$, Wenfeng Xie ${ }^{3}$, Hailin Zhu ${ }^{1}$, Xiaoyan Yin ${ }^{1}$ and Meihui Xu ${ }^{4}$ \\ ${ }^{1}$ School of Information Science \& Engineering, Qilu normal university, 250013, Jinan, China \\ ${ }^{2}$ Presidents' Office, Qilu normal university, 250013, Jinan, China \\ ${ }^{3}$ Office of Educational Administration, Qilu normal university, 250013, Jinan, China \\ ${ }^{4}$ School of Information Science \& Engineering, University of Jinan, 250022, Jinan, China
}

\begin{abstract}
The smart water meter based on cloud computing is a new instrument that uses modern sensor technology, communication technology and modern microelectronics technology to measure water volume, transfer water data and settle transactions. The traditional water meter only has the function of flow collection and pointer mechanical display of water consumption. Compared with traditional water meter, smart water meter has great progress. With the development of wireless transmission technology and mobile communication network, remote water meter technology begins to develop rapidly.
\end{abstract}

\section{Introduction}

For a long time, the water meters we use are generally lowgrade. This kind of instrument has low price and single performance. With the progress of society, people's demand is higher and higher. The shortcomings of traditional water meter are more and more obvious. The traditional meter reading method requires staff to measure and verify the data in person, which consumes a lot of manpower and material resources.

With the rapid development of information technology, the collection and transmission of all kinds of information realize gradually intelligent. The era of using manual doorto-door meter reading and water meter rotary valve to count water consumption will become history. The traditional mechanical water meter could not meet the new business needs. It needs to report the water information timely through the smart water meter. It can sense the turnover state of the urban water supply system in time, and can load the monitoring functions of water quality and water pressure. The smart water meter will become the new development trend of water meter with the application of Internet of things technology, electronic technology, wireless transmission technology, network technology and computer technology. The smart water meter system is based on the Internet of things technology and integrates NB-IOT data transmission module. The system also integrates upper computer software, regional control cabinet, shunt controller and transmitter. It can realize intelligent control through organic combination with water supply system.

Based on the development of smart city, this research proposes a set of reasonable, low-cost and easy-to-use smart water meter system solutions. With the help of cloud computing, the water meter system can realize remote monitoring, data transmission and intelligent control. The smart water meter system is an automatic, digital network management system for water meter reading and water fee solution in water industry.

\section{Overall system architecture}

\subsection{Whole framework}

The platform can transmit accurately the information of multiple distributed units to the integrated system of the next base station. That is the data processing center. The system includes the medium of transmitting information, the high precision instrument of acquiring information and the efficient and fast server etc. The whole framework of the intelligent water meter system is shown in Figure 1.

\subsection{Main functions of the system}

Smart water meter system includes real-time monitoring, equipment support, statistical analysis, historical view, file management and other functional modules. The main function modules are as follows.

1. Data connection.

It can transfer the acquired data to the computer for corresponding data processing.

2. Meter reading management.

Meter reading times and time can be set according to certain rules.

3. File management.

It can measure water meter by category.

4. Real time monitoring.

Users can observe the use of meters at any time. 


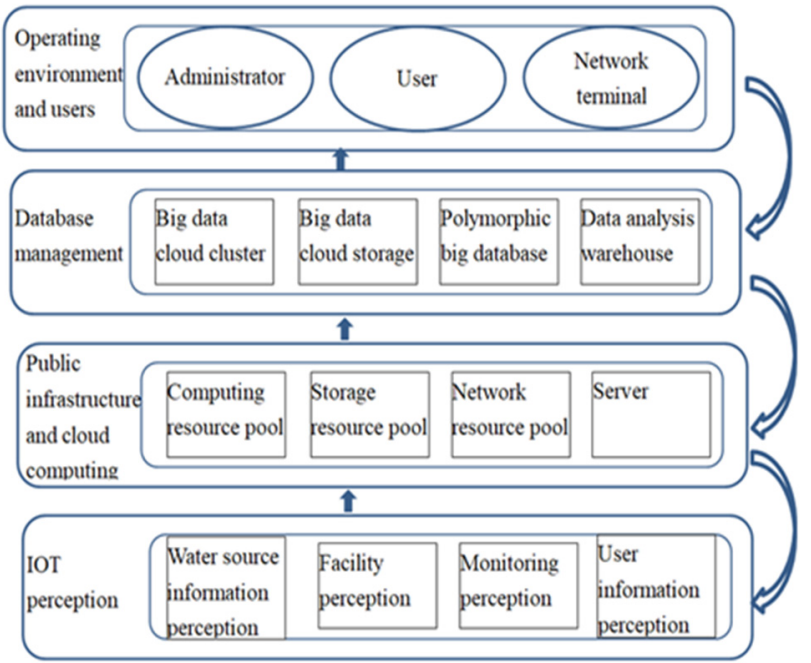

Fig1. The whole framework of the intelligent water meter system

\section{User management}

The user information can be input into the form, and then directly imported into the system. The user ID, name, geographical location and water consumption of the user are concentrated in a table, which is convenient for management and query.

6. System settings.

It can set the administrator's information and related system data.

7. Settlement and billing.

According to the usage, it can settle the user's expenses of each table and print the consumption list. It can also facilitate the user to pay.

8. Historical query.

Historical water consumption can be queried, and the historical water consumption can be exported to save the data for future data analysis.

9. Statistical analysis.

It can carry out statistical analysis of user water consumption according to the date and present it in the form of chart.

The application software structure of smart water meter platform is shown in Figure 2.

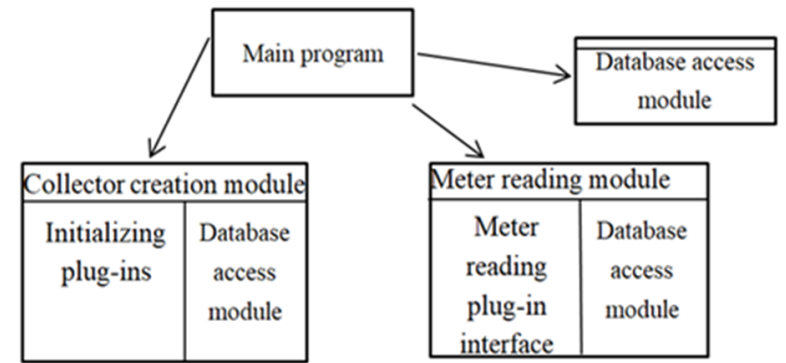

Fig2. The application software structure of smart water meter platform

\section{Equations and mathematics}

Remote meter reading module is the core of the whole smart water meter system. The main function of the remote centralized meter reading module is that the information of each scattered base meter is transmitted to the base station of the general integrated system without error. It includes high-precision instruments to collect information, accurate transmission media and efficient cloud processing computer. Therefore, the remote centralized meter reading system is mainly composed of remote meter reading control valve module, data acquisition module, concentrator and cloud processor.

\subsection{Remote centralized meter reading}

Remote meter reading can realize remote collection of equipment data without substantial contact. The collection process can be carried out automatically, so as to realize the high-frequency data collection, and then master the water used rules more precisely. In order to meet the special needs of different locations, there are usually many different configuration schemes in a system. The mechanism of remote meter reading system requires that all components must be compatible with other communication equipment, and can be extended and upgraded. The network topology of remote centralized meter reading system is shown in Figure 3. At present, Wireless water meter is widely used in youth apartment, white-collar apartment and hotel apartment, which directly connects to remote server. Of course, there are also GPRS water meters, which can be used directly through APP application. The real smart water meter in the future of Internet of things is NB-IOT water meter. It is a smart water meter directly connected to remote server through NB-IOT network.

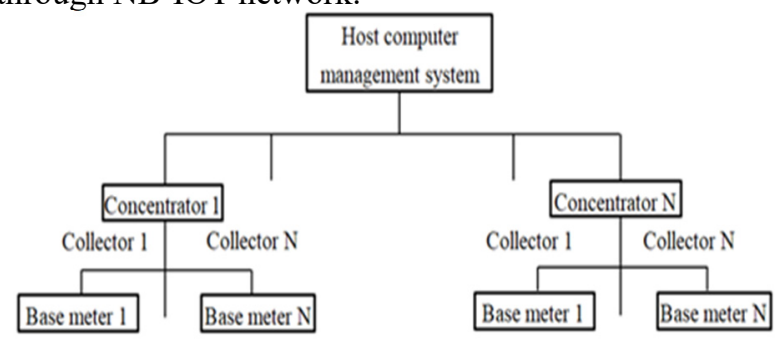

Fig3. Network topology of remote centralized meter reading system

\subsection{System communication mode}

To solve the problem of data communication is the key to solve the remote centralized meter reading. Therefore, the development of various communication methods has also affected the development of remote meter reading. According to the different communication modes, there are mainly wired transmission mode and wireless transmission mode. The wired transmission mode includes bus, power carrier, data network, modem and so on. The wireless transmission mode includes wireless transmission and GSM wireless transmission.

The platform adopts wireless transmission mode and simplifies NB-IOT protocol and signaling process based on LTE technology. In terms of network communication function, NB-IOT network is facing the needs of deep coverage, low power consumption, low cost and mass access of mobile Internet of things. After the adjustment of data transmission scheme, signaling process optimization and mobile management scheme, the network adapts to the data transmission requirements of 
NB-IOT terminal at low speed and low frequency. And it assists the terminal to achieve energy saving and consumption reduction.

\subsection{Key technology NB-IOT}

The wireless communication of smart water meter adopts NB-IOT network. This is shown in Figure 4.The water meter is powered by battery. According to the industry standard, the service life of the battery is not less than six years. NB-IOT technology has great advantages. It has ultra-low power consumption, precise micro power operation module. And it focuses on dual sensing, software error correction, encryption and anti-interference technology. This ensures accurate data transmission. The key technical process of NB-IOT mainly includes: NonIP, data transmission, mobility management, etc.

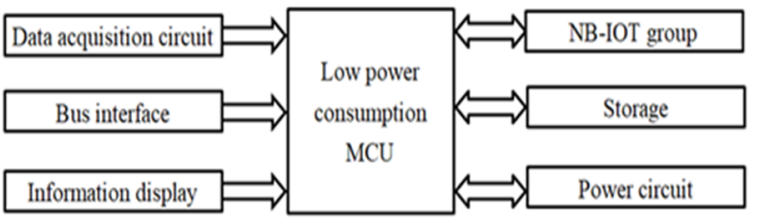

Fig4. Scheme of NB-IOT module combined with water meter

This kind of water meter is composed of the following parts: ultra-low power single chip microcomputer, NB IOT module, data acquisition circuit, information display, power supply circuit, bus interface, storage module.

1. Data acquisition circuit.

It uses analog-to-digital conversion technology to convert the collected information into digital information that can be recognized by the computer.

2. Bus interface.

A general interface is used to transmit the acquisition modules of multiple smart water meters to the upper computer through NB-IOT.

3. Information display.

The water meter information display module adopts LCD output. For low power consumption consideration, the water meter uses LCD display because the power consumption of LCD is far less than LED.

\section{System implementation}

\subsection{System user data query}

After the user fills in the relevant selection information, the database is connected after verification. If the connection is not successful, the relevant page will pop up. If the query is successful, the current information is saved and the program is ended.

\subsection{The system module implemented}

There are many functions in the system. The main functions listed below are water management, user management, recharge and payment records, message push and remote control valve.

1. Water query module.

After logging in, the manager or user will directly display the success rate of meter reading, water meter dosage and analysis.

2. Water management module.

In this module, the administrator can query the water consumption of each household, increase the information of users, edit the daily water consumption of users for control, and generate excel reports. Users can also check their water consumption balance and other information. The water display interface of water meter is shown in Figure 5 .

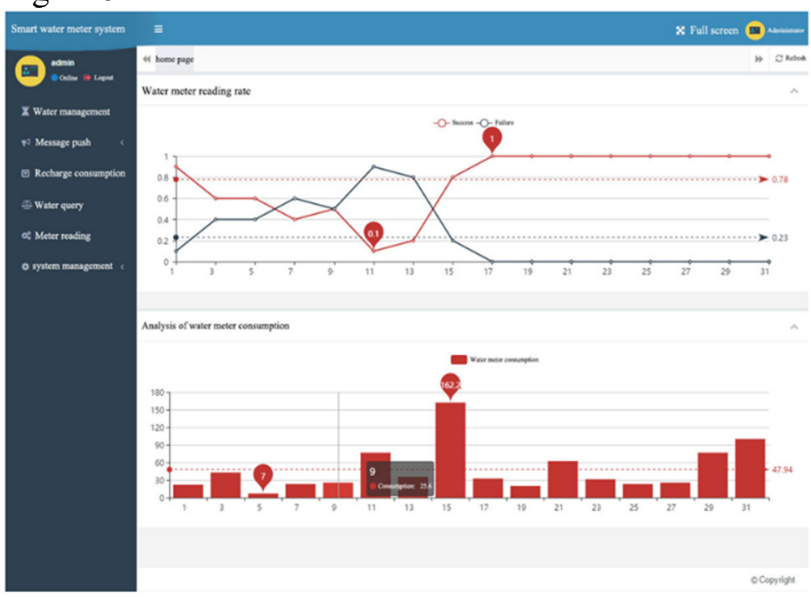

Fig5. Water meter display

3. File management.

This module can retrieve qualified users according to the status of water meter and water consumption time. And it can remotely close the valve of user's water meter.

\subsection{Algorithm implementation}

The smart water meter platform based on cloud computing can initialize data and complete remote meter reading. It can read and save and refresh data. Through algorithm design, it can initialize the tree structure control and its properties to display all user information.It can be copied by clicking on the user tree structure and selecting the corresponding region.It can prompt to save the monthly report and call the stored procedure to create the monthly report and save the call to the background stored procedure.After reading the data, it can refresh the read water meter information. In the aspect of algorithm implementation, remote meter reading module algorithm is more complex. Part of the algorithm implementation is as follows. 


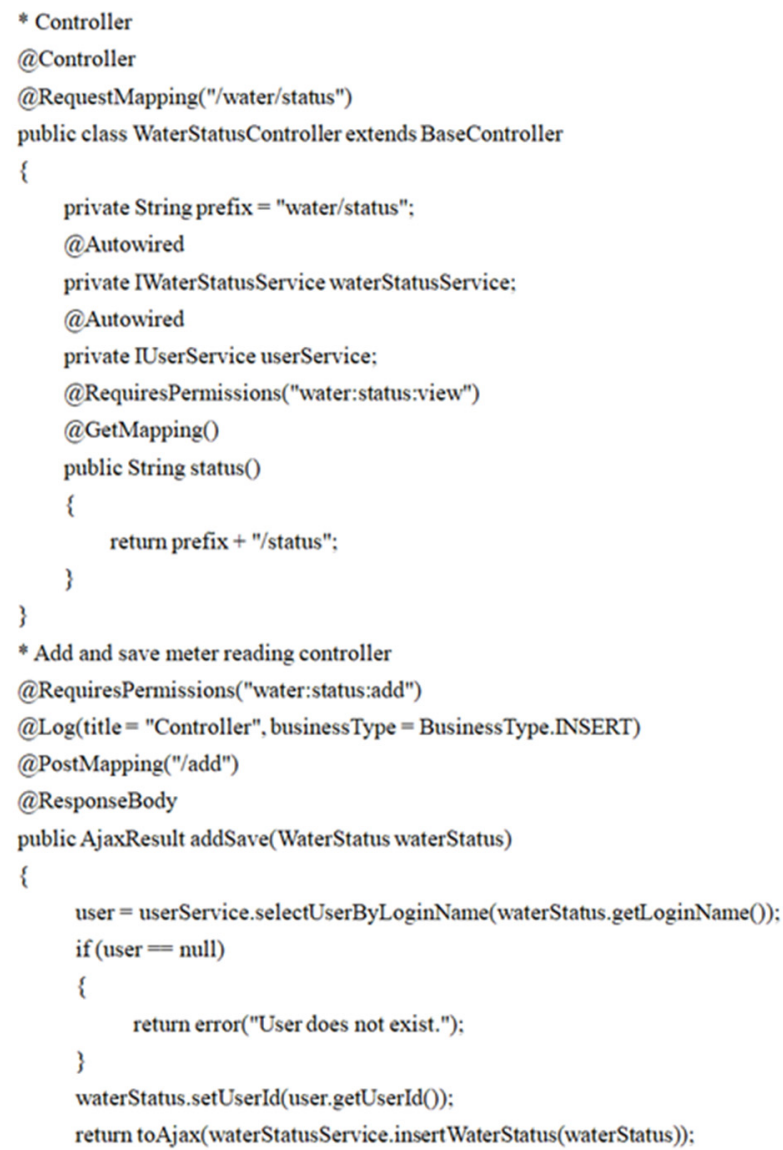

\section{Conclusion and prospect}

This research designs the overall architecture of smart water meter system based on cloud computing. It realizes the system management, remote meter reading, data communication, cloud server processing and other related functions with the help of cloud computing. And it describes the corresponding implementation of algorithms. However, the smart water meter system is a large system. Many aspects need to be further improved, and it will develop towards a more intelligent direction. Using modern internet information technology, measurement sensor internet of things technology, big data and cloud computing application technology, the smart system has high practical value in business optimization and production management.

\section{Acknowledgements}

This work is supported by the Shandong Higher Education Undergraduate Education Reform Project (z2018x067) and the Ministry of Education Cooperative Education Project (201602028018). It is also supported by the Social Science Planning and Research Project of Shandong Province (17CGLJ10).

\section{References}

1. S. Uzayr, N. Cloud,T. Ambler, Vue.js, 1(2019)

2. R. Cobacho, Water meter and measurement (2015)

3. Huang Yuhong, Technical analysis and case analysis of $\mathrm{Nb}$ IOT Internet of things (2019)

4. Fan yuan, Smart city and information security in the era of big data (2019)

5. Sun Guoxiang, On the development and overall design of remote intelligent water meter reading system, Shijiazhuang water supply group (2017)

6. Huang Kai, Cloud computing system and application of artificial intelligence (2014)

7. Liao Zhengwei, Research and practice of intelligent water affairs. (2014)

8. Wen Ying, design and implementation of distributed data mining system based on cloud computing, Electronic technology and software engineering (2016)

9. Wei Feng, Tang Qiang, An urban intelligent water service architecture, computer knowledge and technology (2015)

10. $\mathrm{Ji} \mathrm{Zhu}$, software design of intelligent water integration platform, Hydropower plant automation (2016) 